\title{
$\square+\infty$ \\ GYNECOLOGICAL CANCER \\ Symptom burden and quality of life with chemotherapy for recurrent ovarian cancer: the Gynecologic Cancer InterGroup-Symptom Benefit Study
}

Yeh Chen Lee (D) ,1,2,3 Madeleine T King, ${ }^{4}$ Rachel L O'Connell, ${ }^{2}$ Anne Lanceley, ${ }^{5}$ Florence Joly, 6,7 Felix Hilpert, ${ }^{8,9}$ Alison Davis, ${ }^{1,10}$ Felicia T Roncolato, ${ }^{1,2}$ Aikou Okamoto, ${ }^{11,12}$ Jane Bryce, ${ }^{13,14,15}$ Paul Donnellan, ${ }^{16}$ Amit M Oza, ${ }^{17,18}$ Elisabeth Avall-Lundqvist, ${ }^{19,20,21}$ Jonathan S Berek, ${ }^{22,23}$ Jonathan A Ledermann (D) , ${ }^{24}$ Dominique Berton, ${ }^{6,25}$ Jalid Sehouli, ${ }^{26,27}$ Amanda Feeney, ${ }^{24}$ Marie-Christine Kaminsky, ${ }^{6,28}$ Katrina Diamante, ${ }^{2}$ Martin R Stockler, ${ }^{2}$ Michael L Friedlander (D) , ${ }^{1,3}$ for the GCIG Symptom Benefit Group

- Additional supplemental material is published online only. To view, please visit the journal online (http://dx.doi.org/ 10.1136/ijgc-2021-003142).

For numbered affiliations see end of article.

\section{Correspondence to}

Professor Michael L Friedlander, Prince of Wales Hospital, Barker Street, Randwick, New South Wales 2031, Australia; m. friedlander@unsw.edu.au

Received 26 September 2021 Accepted 7 January 2022

Check for updates

(C) IGCS and ESG0 2022. Re-use permitted under CC BY-NC. No commercial re-use. Published by BMJ.

\begin{tabular}{l}
\hline To cite: Lee YC, King MT, \\
O'Connell RL, et al. Int J \\
Gynecol Cancer Published \\
Online First: [please include \\
Day Month Year]. doi:10.1136/ \\
ijgc-2021-003142 \\
\hline
\end{tabular}

\section{HIGHLIGHTS}

- Most participants reported symptoms related to recurrent ovarian cancer

- $40 \%$ of patients symptomatic at baseline reported an improvement in symptoms within 2 months of starting chemotherapy

- Improvements in quality of life were reported by approximately one in six participants on chemotherapy

\section{ABSTRACT}

Objective The Gynecologic Cancer InterGroup (GCIG)Symptom Benefit Study was designed to evaluate the effects of chemotherapy on symptoms and health-related quality of life (HRQL) in women having chemotherapy for platinum resistant/refractory recurrent ovarian cancer (PRR-ROC) and potentially platinum sensitive with $\geq 3$ lines of chemotherapy (PPS-ROC $\geq 3$ ).

Methods Participants completed the Measure of Ovarian Cancer Symptoms and Treatment (MOST) and European Organization for Research and Treatment of Cancer (EORTC) Quality of Life Questionnaire QLQ-C30 questionnaires at baseline and every 3-4 weeks until progression. Participants were classified symptomatic if they rated $\geq 4$ of 10 in at least one-third of symptoms in the MOST index. Improvement in MOST was defined as two consecutive scores of $\leq 3$ in at least half of the symptomatic items at baseline. Improvement in HRQL was defined as two consecutive scores $\geq 10$ points above baseline in the QLQ-C30 summary score scale (range 0-100).

Results Of 948 participants enrolled, 910 (96\%) completed baseline questionnaires: 546 with PRR-ROC and 364 with PPS-ROC $\geq 3$. The proportions of participants symptomatic at baseline as per MOST indexes were: abdominal $54 \%$, psychological $53 \%$, and disease- or treatment-related $35 \%$. Improvement was reported in MOST indexes: abdominal 40\%, psychological 35\%, and disease- or treatment-related $38 \%$. Median time to improvement in abdominal symptoms occurred earlier for PRR-ROC than for PPS-ROC $\geq 3$ ( 4 vs 6 weeks, $p=0.044$ ); median duration of improvement was also similar (9.0 vs 11.7 weeks, $p=0.65$ ). Progression-free survival was longer among those with improvement in abdominal symptoms than in those without (median 7.2 vs 2.5 months, $\mathrm{p}<0.0001)$. Improvements in HRQL were reported by $77 / 448(17 \%)$ with PRR-ROC and 61/301 (20\%) with
PPS-ROC $\geq 3(p=0.29)$, and $102 / 481(21 \%)$ of those with abdominal symptoms at baseline.

Conclusion Over $50 \%$ of participants reported abdominal and psychological symptoms at baseline. Of those, $40 \%$ reported an improvement within 2 months of starting chemotherapy. Approximately one in six participants reported an improvement in HRQL. Symptom monitoring and supportive care is important as chemotherapy palliated less than half of symptomatic participants.

\section{BACKGROUND}

Patients with platinum resistant/refractory recurrent ovarian cancer (PRR-ROC) and potentially platinum sensitive recurrent ovarian cancer following $\geq 3$ lines of prior chemotherapy (PPS-ROC $\geq 3$ ) often experience a multitude of symptoms related to disease progression and cumulative toxicities from prior chemotherapy. ${ }^{1}$ It can be difficult to distinguish between disease- and chemotherapy-related symptoms such as fatigue, nausea and constipation, among others. Many patients also experience anxiety and depression. ${ }^{2}$ The goals of chemotherapy in this setting include palliating symptoms, improving health-related quality of life (HRQL), and delaying disease progression. Despite decades of chemotherapy trials in recurrent ovarian cancer, there is uncertainty about how well 'palliative' chemotherapy improves symptoms as this is not usually assessed or reported in clinical trials. $^{3}$

Few trials in recurrent ovarian cancer have reported the effects of chemotherapy either on symptoms or on improvements in HRQL. ${ }^{4-7}$ Trials of chemotherapy in PRR-ROC have reported median progression-free 


\section{Original research}

survival of 3-4 months and objective tumor response rates of 4-20\%. Although objective tumor response rates are higher in PPS-ROC $\geq 3$, the likelihood of response and the duration of response decrease with each subsequent line of chemotherapy and are similar to those reported in PRR-ROC. ${ }^{910}$ Given that chemotherapy is meant to palliate symptoms, it is important to document the nature and burden of symptoms at baseline, and to evaluate the impact of chemotherapy on symptoms and HRQL. ${ }^{4}$

Based on data from the Gynecologic Cancer InterGroup (GCIG)Symptom Benefit Study, we have previously reported the predictors of stopping chemotherapy early and survival outcome in patients with PRR-ROC and PPS-ROC $\geq 3 .{ }^{11}{ }^{12}$ We report here on their symptom burden at baseline, rates of improvement in symptoms with chemotherapy, time to improvement of symptoms, and duration of improvement. We also report the overall HRQL at baseline, rates of improvement with chemotherapy, and the correlation with the reported improvement in symptoms. The rationale for the study was to inform discussions with patients and their families about the possible benefits and harms of palliative chemotherapy in recurrent ovarian cancer, and to enable the inclusion of symptom benefit as an endpoint in future clinical trials.

\section{METHODS}

\section{GCIG-Symptom Benefit Study}

The GCIG-Symptom Benefit Study (clinical trial registration number ACTRN12607000603415, Online supplemental file 1) was a


Stage 2 aimed to determine the proportion of women benefiting from palliative chemotherapy as defined by a clinically significant improvement in HRQL scores and improvement of symptoms. Participants had either PRR-ROC or PPS-ROC $\geq 3$ and had been recommended palliative chemotherapy by their treating clinician. ${ }^{13}$ The study was in accordance with the NHMRC Statement on Ethical Conduct in Research Involving Humans and the Declaration of Helsinki, with ethics approval at all participating sites and signed written informed consent was obtained from all participants.

Symptoms and aspects of HRQL were assessed with the Measure of Ovarian cancer Symptoms and Treatment (MOST-T35), ${ }^{13}{ }^{14}$ European Organization for Research and Treatment of Cancer (EORTC) Quality of Life Questionnaire QLQ-C30, ${ }^{16}{ }^{16}$ QLQ-OV28, ${ }^{17}$ Functional Assessment of Cancer Therapy-Ovarian (FACT-0), ${ }^{18}$ and Functional Assessment of Cancer Therapy-Ovarian Symptom Index (FOSI). ${ }^{19}$ Participants completed these questionnaires without assistance on paper while waiting to see their physicians at baseline and before each cycle of chemotherapy until disease progression. Participants were included in the analysis if they completed a questionnaire at baseline and at least one subsequent time point.

\section{Symptom Assessment with MOST-T35}

Symptoms and well-being were assessed with MOST-T35, ${ }^{14}$ which includes 35 items. Three items assess well-being (physical, emotional, overall) on a numeric rating scale from 0 (worst possible) to 10 (best possible). The remainder assess symptoms and treatment-related difficulties on a rating scale from 0 (none at all) to 10 (worst I can imagine), with additional verbal anchors for mild (1-3), moderate (4-6), and severe (7-9). Twenty-four of these items were grouped into five indexes: abdominal (MOST-Abdo,
2 symptoms), disease- or treatment-related (MOST-DorT, 11 symptoms), chemotherapy-related (MOST-Chemo, 6 symptoms), psychological (MOST-Psych, 2 symptoms), and well-being (MOSTWell-being, 3 items). ${ }^{13}$ Index scores were calculated as the average of the component items, linearly transformed to give a score from 0 (none at all) to 100 (most severe) for the four symptom indexes and 0 (worst possible) to 100 (best possible) for the well-being index. $^{13}$

Patients were categorized as symptomatic at baseline if they scored $\geq 4$ out of 10 (verbal anchors of moderate, severe, and worst I can imagine) for at least one-third of the items in a MOST symptom index: 1 of 2 items for MOST-Abdo and MOST-Psych, 4 of 11 items for MOST-DorT, and 2 of 6 items for MOST-Chemo. Symptom improvement was defined as $\geq 2$ consecutive scores of $\leq 3$ in items that were symptomatic at baseline and required at least two symptoms for MOST-Chemo and MOST-DorT and at least one symptom for MOST-Abdo and MOST-Psych to improve. For the MOST-Well-being index, a score of $\leq 6$ in at least one well-being item at baseline was considered to represent impaired well-being, and improvement was defined as a score of $\geq 7$ in at least one of the items that scored $\leq 6$ at baseline.

\section{HRQL Assessment with EORTC QLQ-C30 and QLU-C10D}

Aspects of $H R Q L$ were measured with the EORTC QLQ-C30 which includes 30 items that collectively assess five aspects of functioning (physical, role, cognitive, emotional, and social functioning), eight symptoms commonly experienced by cancer patients, financial difficulties, and a global rating of health and quality of life. ${ }^{15} 16$

In this analysis we took two approaches to scoring data from the QLQ-C30 to provide a comprehensive measure of aspects of HRQL. The first was to use the QLQ-C30 summary score, which incorporates 27 items (excluding three items: financial difficulties, global quality of life, and health). ${ }^{20}{ }^{21} \mathrm{~A}$ higher score of QLQC30 summary score indicates a better HRQL. An improvement in overall HRQL was defined as two or more consecutive summary scores that were $\geq 10$ points higher than the summary score at baseline.

The second approach was to use the EORTC QLU-C10D utility score which incorporates 13 items from the QLQ-C30 covering physical, emotional, role, and social functioning, plus symptoms often experienced by patients with ovarian cancer (fatigue, pain, nausea, sleep problems, bowel problems, and appetite loss). ${ }^{22}$ Validated Australian utility weights were used, resulting in a score from -0.095 (worst) to 1 (best). ${ }^{23}$ An improvement in the QLU-C10D utility score was defined as $\geq 2$ consecutive scores that were $\geq 0.10$ higher than the score at baseline.

\section{Statistical Analysis}

Continuous variables are reported as mean or median (IQR). Counts with percentages are reported for categorical variables. The $\chi^{2}$ test was used to compare proportions and the Wilcoxon rank-sum test was used for comparisons of non-normally distributed continuous variables. We used the Kaplan-Meier method and log rank test to analyze progression-free survival. Statistical inferences were based on a two-sided significance level of 0.05 without correction for multiplicity. Analyses were performed with SAS version 9.4 and $R$ version 3.6.3. 
Table 1 Numbers of participants completing patient-reported outcome measures (PROMs) at each time point

\begin{tabular}{|c|c|c|c|c|c|c|c|}
\hline Time point & Baseline & Pre-C2 & Pre-C3 & Pre-C4 & Pre-C5 & Pre-C6 & Pre-C7 \\
\hline $\begin{array}{l}\text { PROM } \\
\text { completed/ } \\
\text { number } \\
\text { proceeding with } \\
\text { chemotherapy* } \\
\text { (\%) }\end{array}$ & 910/933 (98\%) & $765 / 838(91 \%)$ & $611 / 673(91 \%)$ & $452 / 499(91 \%)$ & $361 / 406(89 \%)$ & $250 / 286(87 \%)$ & $126 / 150(84 \%)$ \\
\hline $\begin{array}{l}\text { PROM } \\
\text { completed but } \\
\text { chemotherapy } \\
\text { discontinued }\end{array}$ & 0 & 29 & 75 & 101 & 65 & 113 & 159 \\
\hline \multicolumn{8}{|c|}{ PROM completed according to treatment group } \\
\hline PRR-ROC, $\mathrm{n}_{1}$ & 546 & 461 & 390 & 293 & 222 & 189 & 136 \\
\hline $\begin{array}{l}\text { PPS-ROC } \geq 3, \\
n_{2}\end{array}$ & 364 & 333 & 296 & 260 & 204 & 174 & 149 \\
\hline
\end{tabular}

${ }^{*}$ Number of participants who completed PROM questionnaires divided by number of participants proceeding with chemotherapy. $\mathrm{C}$, chemotherapy cycle; PPS-ROC $\geq 3$, potentially platinum sensitive recurrent ovarian cancer after $\geq 3$ lines of chemotherapy; PROM, patient reported outcome measure; PRR-ROC, platinum resistant/refractory recurrent ovarian cancer.

\section{RESULTS}

We recruited 948 participants to GCIG-Symptom Benefit Study stage 2, of whom $933(98 \%)$ received at least one cycle of chemotherapy. This study was an expansion of stage 1 , which reported a high symptom burden in 126 participants with PRR-ROC at baseline, of which almost $50 \%$ of symptomatic participants reported symptom improvement. ${ }^{1}$ Baseline questionnaires were completed by 910 of $933(98 \%)$ patients: $546(60 \%)$ with PRR-ROC and $364(40 \%)$ with PPS-ROC $\geq 3$. Questionnaire completion rates (number who completed questionnaires divided by number continuing chemotherapy) were $84 \%$ or higher at all time points (Table 1). Patients who ended the chemotherapy cycle but continued to complete questionnaires are also shown in Table 1. Six or more cycles of chemotherapy were administered in $28 \%$ (162/570) of those with PRR-ROC and $33 \%$ (124/379) of those with PPS-ROC $\geq 3$.

\section{Baseline Symptom Burden and Symptom Improvement on Chemotherapy According to MOST Indexes}

The numbers and proportions of women classified as symptomatic at baseline according to the five MOST indexes, the number showing an improvement in symptoms and well-being, and the time to improvement in symptoms are shown in Table 2 for all participants and for the sub-groups with PRR-ROC and PPS-ROC $\geq 3$.

At baseline, before starting chemotherapy, approximately half the participants were classified as symptomatic according to the MOST-Abdo index (481/893, 54\%) or MOST-Psych index (472/896, $53 \%)$, one-third according to the MOST-DorT index (314/903, 35\%), and one-quarter according to the MOST-Chemo index (244/898, $27 \%$ ). Approximately three-quarters of all participants were classified as having impaired well-being at baseline $(633 / 883,72 \%)$. These proportions were similar among those with PRR-ROC and those with PPS-ROC $\geq 3$ (all $p>0.16$ ).

Among participants classified as symptomatic at baseline, improvements during chemotherapy were observed in approximately $40 \%$ according to MOST-Abdo (190/481, 40\%) and MOST-DorT (118/314, 38\%), one-third according to MOST-Psych (165/472, 35\%), and one-quarter according to MOST-Chemo
(68/244, 28\%) and MOST-Well-being (156/633, 25\%). Improvements occurred with similar frequencies among those with PRR$\mathrm{ROC}$ and PPS-ROC $\geq 3$.

The median time to improvement in symptoms ranged from 4 to 7 weeks. Improvements in MOST-Abdo occurred earlier for those with PRR-ROC than PPS-ROC $\geq 3$ (median 4 vs 6 weeks, $p=0.044$ ). The median time to improvement in MOST-Well-being was $5-6$ weeks. The median duration of improvement in MOST-Abdo symptoms among all participants was 10 weeks (IQR 4-20) and was similar among those with PRR-ROC and those with PPS-ROC $\geq 3$ ( 9.0 vs 11.7 weeks, $p=0.65$ ).

Progression-free survival was longer among participants with abdominal symptoms at baseline that improved than in those with abdominal symptoms that did not improve (median 7.2 vs 2.5 months, $p<0.0001$; Figure 1). Participants without abdominal symptoms at baseline had a median progression-free survival of 5.3 months.

\section{HRQL Improvement According to the EORTC QLQ-C30 Summary Score and QLU-C10D Utility Score}

At baseline, before starting chemotherapy, the proportion of participants who reported reduced HRQL (EORTC QLQ-C30 summary score $\leq 90$ points on a range from 0 to 100) was similar for PRRROC (448/516, 87\%) and PPS-ROC $\geq 3(301 / 349,86 \%)$. Improvements in HRQL were recorded with similar frequency among those with PRR-ROC and those with PPS-ROC $\geq 3(77 / 448(17 \%)$ vs $61 / 301(20 \%), p=0.29)$. The median time to improvement in summary score for HRQL was 5 weeks and 9 weeks, respectively $(p=0.06)$.

In general, QLQ-C30 summary scores were higher among participants who completed a larger number of assessments, and summary scores declined as participants approached their last assessments (Figure 2). At baseline, a QLU-C10D score $\leq 0.9$ (on a scale from -0.095 to 1.0 ) was reported by the same proportions of participants with PRR-ROC (469/509, 92\%) and PPS-ROC $\geq 3$ (313/342, 92\%). An improvement in HRQL according to the QLUC10D was reported by fewer participants with PRR-ROC than PPS-ROC $\geq 3$ (90/469 (19\%) vs $82 / 313(26 \%), p=0.02)$. Time to improvement in the QLU-C10D score was somewhat shorter among 
Table 2 Burden at baseline and subsequent improvements in symptoms and well-being assessed with MOST index scores

\begin{tabular}{|c|c|c|c|c|c|c|c|}
\hline \multirow[b]{2}{*}{ MOST indexes } & \multicolumn{2}{|c|}{ All participants } & \multicolumn{2}{|c|}{ PRR-ROC } & \multicolumn{2}{|c|}{ PPS-ROC $\geq 3$} & \multirow[b]{2}{*}{$\begin{array}{l}P \text { value* } n_{1} \text { vs } \\
n_{2}\end{array}$} \\
\hline & $\mathbf{N}_{0}$ & $\begin{array}{l}\text { Symptomatic at } \\
\text { baseline, } n_{0}(\%)\end{array}$ & $\mathbf{N}_{1}$ & $\begin{array}{l}\text { Symptomatic at } \\
\text { baseline, } n_{1}(\%)\end{array}$ & $\mathbf{N}_{2}$ & $\begin{array}{l}\text { Symptomatic at } \\
\text { baseline, } n_{2}(\%)\end{array}$ & \\
\hline MOST-Psych $\ddagger$ & 896 & $472(53)$ & 534 & $278(52)$ & 362 & $194(54)$ & 0.65 \\
\hline MOST-DorT§ & 903 & $314(35)$ & 540 & $192(36)$ & 363 & $122(34)$ & 0.55 \\
\hline $\begin{array}{l}\text { MOST-Well- } \\
\text { being }\end{array}$ & $\mathbf{N}_{0}$ & $\begin{array}{l}\text { Symptom } \\
\text { improvement, } n_{0}(\%)\end{array}$ & $N_{1}$ & $\begin{array}{l}\text { Symptom } \\
\text { improvement, } n_{1}(\%)\end{array}$ & $\mathbf{N}_{2}$ & $\begin{array}{l}\text { Symptom } \\
\text { improvement, } n_{2}(\%)\end{array}$ & $\begin{array}{l}P \text { value }{ }^{*} n_{1} \text { vs } \\
n_{2}\end{array}$ \\
\hline MOST-Abdo & 481 & $190(40)$ & 296 & $107(36)$ & 185 & $83(45)$ & 0.06 \\
\hline \multirow[t]{2}{*}{ MOST-Well-being } & 633 & $156(25)$ & 377 & $85(23)$ & 256 & $71(28)$ & 0.14 \\
\hline & $\mathbf{N}_{0}$ & $\begin{array}{l}\text { Time to improvement, } \\
\text { median (IQR) weeks }\end{array}$ & $\mathrm{N}_{1}$ & $\begin{array}{l}\text { Time to improvement, } \\
\text { median (IQR) weeks }\end{array}$ & $\mathrm{N}_{2}$ & $\begin{array}{l}\text { Time to } \\
\text { improvement, } \\
\text { median (IQR) weeks }\end{array}$ & $\mathbf{P}$ valuet† \\
\hline MOST-Abdo & 190 & $5(4-9)$ & 107 & $4(4-9)$ & 83 & $6(4-12)$ & 0.04 \\
\hline MOST-Psych & 165 & $4(4-8)$ & 93 & $4(4-6)$ & 72 & $5(4-14)$ & 0.26 \\
\hline MOST-DorT & 118 & $5(4-9)$ & 66 & $5(4-8)$ & 52 & $5(4-10)$ & 0.57 \\
\hline MOST-Chemo & 68 & $5(4-13)$ & 50 & $5(4-13)$ & 18 & $7(4-13)$ & 0.54 \\
\hline MOST-Well-being & 156 & $6(4-12)$ & 85 & $5(4-11)$ & 71 & $6(4-16)$ & 0.15 \\
\hline
\end{tabular}

${ }^{*} \chi^{2}$ test.

†MOST-Abdo, 2 symptoms: abdominal pain, discomfort and/or cramps; abdominal swelling, bloating and/or fullness.

‡MOST-Psych, 2 symptoms: anxiety (feeling worried); depression (feeling sad).

§MOST-DorT, 11 symptoms: fatigue (tiredness); trouble eating; indigestion; nausea; vomiting; diarrhea; constipation; shortness of breath; difficulty swallowing; trouble sleeping; bladder problems.

१MOST-Chemo, 6 symptoms: altered sense of taste; sore mouth or throat; hair loss; skin rash; numbness or pins and needles; sore hands and feet.

${ }^{* *}$ MOST-Well-being, 3 items: physical well-being, emotional well-being, overall well-being.

††Wilcoxon rank-sum test.

IQR, interquartile range; MOST, Measure of Ovarian Cancer Symptoms and Treatment; $n$, number; N, denominator; PPS-ROC $\geq 3$, potentially platinum sensitive recurrent ovarian cancer after $\geq 3$ lines of chemotherapy; PRR-ROC, platinum resistant/refractory recurrent ovarian cancer.

those with PRR-ROC than in those with PPS-ROC $\geq 3$ (median 4 vs 7 weeks, $\mathrm{p}=0.02)$.

\section{Improvements in Abdominal Symptoms and HRQL}

The frequencies of abdominal symptoms, improvements in abdominal symptoms, and improvements in HRQL are shown as an alluvial plot in Figure 3. Approximately half the participants were classified as having abdominal symptoms at baseline according to MOST-Abdo (481/893, 54\%). Of those 481 participants, $190(40 \%)$ had an improvement in abdominal symptoms and $102(21 \%)$ had an improvement in HRQL according to the EORTC-QLQ C30 summary score. Among 291 participants with abdominal symptoms at baseline that did not improve, an improvement in HRQL was observed in $21(7 \%)$ participants. An improvement in HRQL was observed in 33 of $412(8 \%)$ participants without abdominal symptoms.

\section{DISCUSSION}

\section{Summary of Main Results}

Our findings confirmed that patients with PRR-ROC and PPS$\mathrm{ROC} \geq 3$ had a high burden of symptoms related to their disease and chemotherapy. Symptom burden at baseline was high and similar among those with PRR-ROC and PPS-ROC $\geq 3$, with over $50 \%$ reporting moderate to severe abdominal symptoms (MOST-Abdo) and psychological symptoms (MOST-Psych). Of those symptomatic at baseline, less than $40 \%$ reported an improvement in symptoms with chemotherapy. Overall, approximately $15 \%$ of participants reported an improvement in aspects of HRQL with chemotherapy. These findings are sobering and demonstrate that only a relatively small proportion of patients overall experience improvement in their symptoms and quality of life as a result of palliative chemotherapy. 


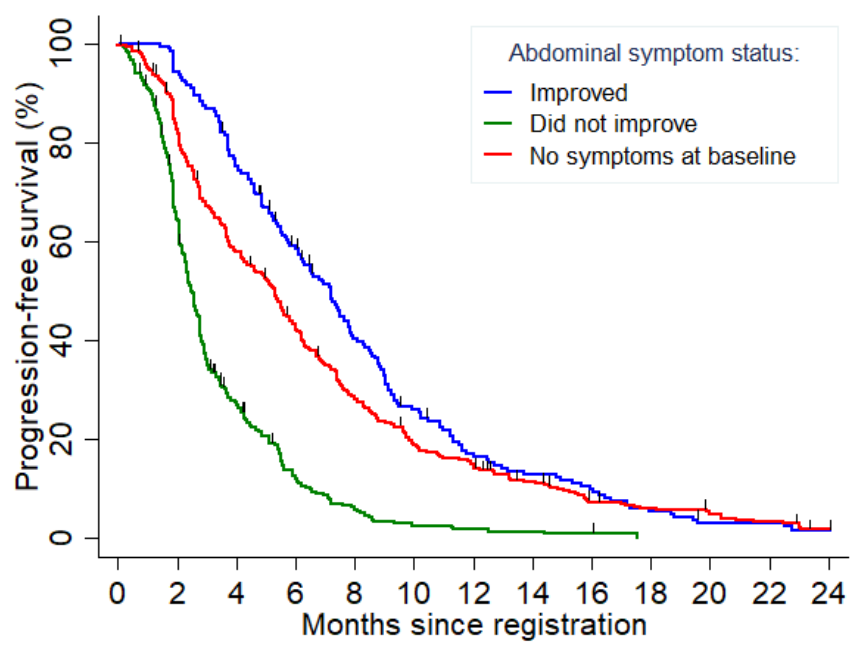

Number at Risk:

Abdominal symptom status:

$\begin{array}{llllllllllllll}\text { Improved } & 190 & 179 & 142 & 105 & 70 & 44 & 28 & 21 & 16 & 9 & 4 & 4 & 2\end{array}$

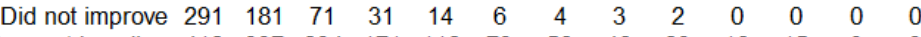

No symptoms at baseline $\begin{array}{llllllllllllll}412 & 337 & 234 & 171 & 112 & 73 & 58 & 40 & 23 & 18 & 15 & 9 & 3\end{array}$

Figure 1 Progression-free survival for sub-groups with symptoms at baseline that improved, symptoms at baseline that did not improve, and no symptoms at baseline.

\section{Results in the Context of Published Literature}

Our findings underscore the importance of assessing symptoms at baseline and during chemotherapy. Similar to PRR-ROC, participants with PPS-ROC $\geq 3$ were found to have a high symptom burden at baseline and marginally higher numerical improvement in symptoms with chemotherapy. Importantly, relative to patients with one or two lines of treatment, our findings showed that the benefit of chemotherapy was low in those who had $\geq 3$ lines of chemotherapy despite being considered to be potentially platinum sensitive, which is in keeping with the clinical observation in routine practice as well as a study reported by Hanker et al. ${ }^{10}$ Our findings also highlight the need to temper patient expectations about the likelihood that symptoms and quality of life will improve with chemotherapy.

The goals of treatment in PRR-ROC and PPS-ROC $\geq 3$ include palliation of symptoms and delaying cancer progression; our results indicate the limited capacity for chemotherapy to achieve

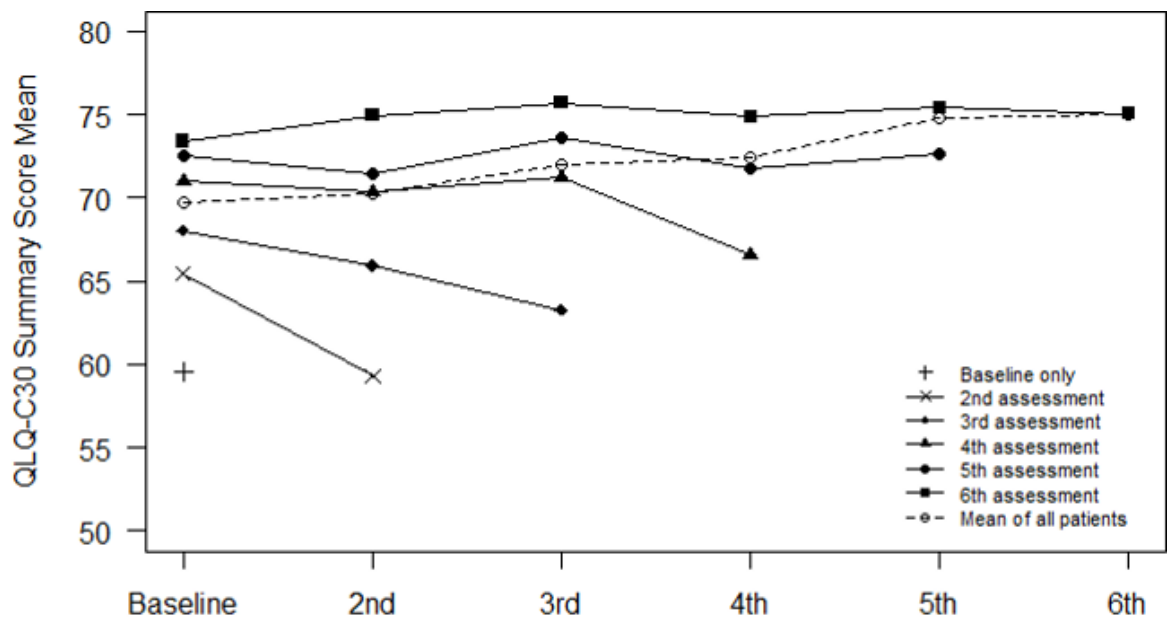

No. patients


135
115
67
312
629

122
71
311
504

80
303
383

Figure 2 European Organization for Research and Treatment of Cancer (EORTC) Quality of Life Questionnaire QLQ-C30 summary scores for overall quality of life by the number of assessment time points at which questionnaires were completed. 


\section{Original research}

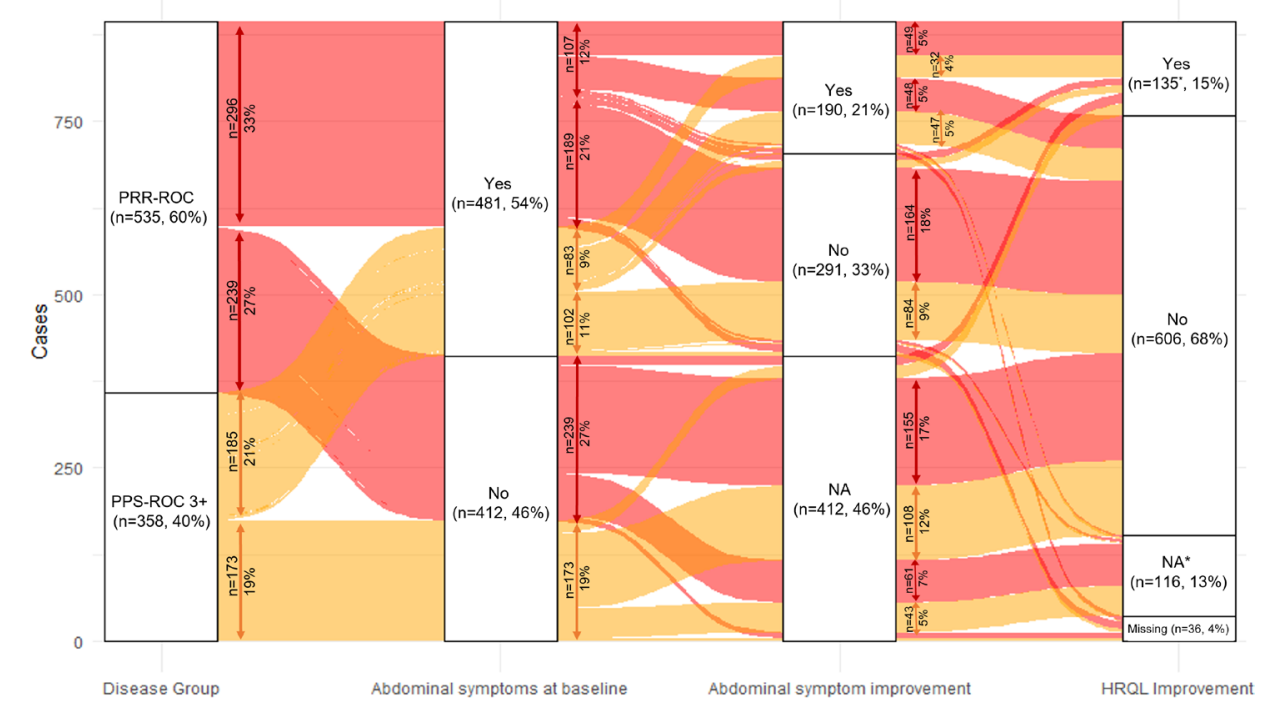

Figure 3 Improvements in abdominal symptoms and overall health-related quality of life (European Organization for Research and Treatment of Cancer (EORTC) Quality of Life Questionnaire QLQ-C30 summary score) according to treatment group and the presence of abdominal symptoms at baseline.

these goals and therefore the importance of supportive care to manage symptoms. We recommend a more comprehensive holistic approach to management and supportive care, including early and routine integration of palliative care, psycho-oncology, and allied health services to address physical, psychological, social, and existential concerns. Moreover, patients' preferences should be included in the treatment decision-making process. ${ }^{24}$ Greater attention is also warranted to address side effects of chemotherapy. ${ }^{25} 26$

Improvements in symptoms and/or HRQL were generally evident within 4-6 weeks (2-3 cycles of chemotherapy). Those with abdominal symptoms at baseline that improved during chemotherapy had a median duration of symptom improvement of 10 weeks and median progression-free survival was 7 months, which is likely a reflection of chemotherapy effect. For the $25 \%$ of participants who reported an improvement in MOST-Well-being, the median time to improvement was 6 weeks. Translating these findings into clinical practice, improvements in symptoms and/or HRQL are likely to be observed within 2 months of starting chemotherapy. Persistence of symptoms after 2 months of chemotherapy for PRR-ROC and PPS-ROC $\geq 3$ should trigger reassessment of disease status and careful consideration of whether to continue chemotherapy, increase palliative care involvement, and discuss advanced care planning.

Many patients will persevere through disease-related symptoms and treatment-related side effects in the hope of a tumor response or delayed progression and longer survival. In reality, response rates reported for single agent chemotherapy in PRR-ROC range from $4 \%$ to $15 \%$ and the impact on overall survival is unclear. ${ }^{8}$ This study showed that regular assessment of symptoms with MOST could help reveal trends in symptoms and well-being that could complement tumor markers and imaging as indicators of treatment benefit. In addition, using MOST would provide a patient-rated measure of chemotherapy toxicities to counter the well-documented underreporting of toxicities inherent in clinicians' assessments. ${ }^{127} 28$

\section{Strengths and Weaknesses of Study}

This study describes the impact of palliative chemotherapy on symptoms and HRQL in a large international real-world patient sample using a dataset of patient-rated outcome measures with excellent $(\geq 84 \%)$ completion rates. Our study demonstrates the importance of regular assessment of symptoms during chemotherapy and underscores the critical importance of supportive care and symptom management beyond the administration of chemotherapy alone. One limitation of our study was that our patientreported outcome data were collected for research and not relayed to the treating clinicians in real time.

\section{Implications for Practice and Future Research}

Future research should involve systematic collection of patientreported outcome measures and real-time reporting to their clinicians, as this can improve patient-clinician communication, responsiveness of clinicians to patients' individual care needs, and patients' understanding and awareness of symptoms. ${ }^{29}{ }^{30} \mathrm{~A}$ systematic review of 22 randomized trials found that proactive use of patient-reported outcome measures in clinical care can be beneficial, particularly when the results were provided to treating clinicians in real time ${ }^{293132}$ but these are not beneficial in all contexts. One of the most influential trials reported improvements in HRQL and overall survival using self-ratings of 12 symptoms commonly experienced during chemotherapy, ${ }^{31}{ }^{33}$ including nine of the symptoms assessed by MOST. Timely and tailored symptom management of patients receiving chemotherapy for ovarian cancer could improve patients' tolerance of chemotherapy.

MOST is an instrument tailored to symptoms and concerns pertinent to ovarian cancer and its treatment. Accordingly, we propose MOST as a suitable candidate for integration into the care of women having treatment for recurrent ovarian cancer, with results reviewed by clinicians in real time. This could improve patients' understanding and encourage them to report key symptoms that occur between clinic visits. Future research in this area is needed, 
and in line with a position statement by the GCIG-Symptom Benefit Committee urging the incorporation of patient-centered benefits as end points for trials in ovarian cancer. ${ }^{34}$

\section{CONCLUSION}

Improvement in symptoms was seen in approximately $40 \%$ of those with moderate or severe symptoms at baseline, with a median time to improvement of $<2$ months. However, many participants did not experience a meaningful improvement in symptoms during chemotherapy. Our findings indicate the need to reassess disease status and decide whether to continue treatment, particularly if symptoms persist beyond 2 months on chemotherapy. In addition, there is a role for using patient-reported outcome measures routinely in clinic to assess symptom benefit during chemotherapy for recurrent ovarian cancer, and the importance of supportive care in this setting.

\section{Author affiliations}

${ }^{1}$ Australia New Zealand Gynaecological Oncology Group (ANZGOG), Camperdown, New South Wales, Australia

${ }^{2}$ University of Sydney, NHMRC Clinical Trials Centre, Camperdown, New South Wales, Australia

${ }^{3}$ Prince of Wales Clinical School, Faculty of Medicine and Health, University of New South Wales, Randwick, New South Wales, Australia

${ }^{4}$ University of Sydney, Quality of Life Office of Psycho-Oncology Research Group (PoCoG), Camperdown, New South Wales, Australia

${ }^{5}$ UCL Elizabeth Garrett Anderson Institute for Women's Health, University College London, London, UK

${ }^{6}$ GINECO-Group d'Investigateurs Nationaux pour l'Etude des Cancers Ovariens, Paris, France

${ }^{7}$ Oncology, Ctr Francois Baclesse, Caen, France

${ }^{8}$ Arbeitsgesmeinschaft Gynäkologische Onkologie Studiengruppe (AGO) und NorthEastern German Society of Gynecologcial Oncology (NOGGO), Kiel, Germany

${ }^{9}$ Onkologisches Therapiezentrum, Krankenhaus Jerusalem, Hamburg, Germany

${ }^{10}$ Medical Oncology, Canberra Hospital, Canberra, Australian Capital Territory, Australia

${ }^{11}$ Japanese Gynecologic Oncology Group (JGOG), Tokyo, Japan

${ }^{12}$ Department of Obstetrics and Gynecology, Jikei University School of Medicine, Minato-ku, Japan

${ }^{13}$ Istituto Nazionale Tumori IRCCS Fondazione Pascale, Napoli, Campania, Italy

${ }^{14}$ Ascension St John Clinical Research Institute, Tulsa, Oklahoma, USA

${ }^{15}$ MITO Multicentre Italian Trials in Ovarian and gynecologic cancer, Italy

${ }^{16}$ Cancer Trials Ireland, Galway University Hospital, Galway, Ireland

${ }^{17}$ Princess Margaret Consortium (PMHC), Toronto, Ontario, Canada

${ }^{18}$ Princess Margaret Cancer Centre, University of Toronto, Toronto, Ontario, Canada

${ }^{19}$ Nordic Society of Gynaecological Oncology (NSGO), Copenhagen, Denmark

${ }^{20}$ Department of Oncology and Department of Clinical and Experimental Medicine,

Linkoping University, Linkoping, Sweden

${ }^{21}$ Department of Oncology-Pathology, Karolinska Institutet, Stockholm, Sweden

${ }^{22}$ Cooperative Gynecologic Oncology Investigators (COGI), Stanford, California, USA

${ }^{23}$ Stanford Women's Cancer Centre, Stanford Cancer Institute, Stanford University School of Medicine, Stanford, California, USA

${ }^{24}$ The Cancer Research UK and UCL Cancer Trials Centre, NCRI UK, London, UK

${ }^{25}$ Institut de Cancérologie de l'Ouest, Centre René Gauducheau, Saint Herblain, France

${ }^{26}$ Arbeitsgesmeinschaft Gynäkologische Onkologie Studiengruppe (AGO) und NorthEastern German Society of Gynecological Oncology (NOGGO), Berlin, Germany

${ }^{27}$ Department of Gynecology and Oncological Surgery, Charité Universitätsmedizin Berlin, Berlin, Germany

${ }^{28}$ Institut de Cancérologie de Lorraine, Vandoeuvre Les Nancy, France

Twitter Yeh Chen Lee @YChenLee

Acknowledgements The Gynecologic Cancer Intergroup (GCIG) Symptom Benefit study was led by the Australia New Zealand Gynaecological Oncology Group
(ANZGOG) in collaboration with the NHMRC Clinical Trials Centre, University of Sydney, Cancer Research UK and UCL Cancer Trials Centre. The authors thank all of the patients and their families, physicians, and nurses who participated in the study.

Contributors YCL, MTK, RO, and MRS contributed to the research proposal, concept development, data analysis, interpretation of results, and drafted and revised the manuscript. $A D$ and FTR contributed to data analysis, interpretation of results and revision of the manuscript. AL, FJ, FH, A0, JB, PD, AMO, EA-L, JSB, $\mathrm{JL}, \mathrm{DB}, \mathrm{JS}, \mathrm{AF}$, and M-CK contributed to patient recruitment to the study, data analysis, interpretation of results and revisions of the manuscript. KD contributed to data acquisition and management, interpretation of results and revised the manuscript. MLF contributed to the research proposal, concept development, patient recruitment to the study, data analysis, interpretation of results, and drafted and revised the manuscript. YCL, MRS, and MLF accept full responsibility for the work and/or the conduct of the study, had access to the data, and controlled the decision to publish.

Funding In Australia the study was supported by NHMRC grants (1063012 and 570893). In the UK this was a National Institute for Health Research (NIHR) study jointly funded by Target Ovarian Cancer (UCL-P001AL) and the Cancer Research UK and UCL Cancer Trials Centre (Programme Grant C444/A15953). MTK is supported by the Australian Government through Cancer Australia. MLF is supported by an NHMRC Program grant. AL was assisted by the National Institute for Health Research (NIHR) UCLH/UCL Biomedical Research Centre which is supported by the Department of Health.

Competing interests YCL has received honoraria from GSK for participation on the Advisory Board. FJ acknowledges institutional research grants from GSK and has received honoraria for educational events from AstraZeneca, Astellas, Amgen, BMS, Bayer, Clovis, GSK, Ipsen, MSD, Janssen, Pfizer, and Sanofi. A0 acknowledges institutional research grants from MSD K.K., Fuji Pharma, Kissei Pharmaceutical, Meiji Holdings, Taiho Pharmaceutical, Kaken Pharmaceutical, Chugai Pharmaceutical, Tsumura, Daiichi Sankyo, Shinnihonseiyaku, Mochida Pharmaceutical, CMIC Holdings, CMIC Holdings, ASKA Pharmaceutical, Takeda, Pfizer, AstraZeneca, and Terumo; and has received honoraria for educational events from Takeda and AstraZeneca. JL acknowledges institutional research grants from AstraZeneca and MSD/Merck and has received honoraria for educational events from AstraZeneca, Neopharm, GSK and Pfizer and participated on the data safety monitoring board of Regeneron. JS has received honoraria (educational events/travel) from AstraZeneca, Clovis, Clovis and Roche; and has participated on the data safety monitoring board or advisory board for AstraZeneca, Clovis, Eisei, GSK, Novocure, Pfizer, Roche, and Tesaro. MLF acknowledges institutional research grants from AstraZeneca, Novartis, and Beigene; has received honoraria (consultancy/educational events) from AstraZeneca, Novartis, GSK, MSD, Takeda, Lily, and ACT-Genomics; and participated on the data safety monitoring board for AGITG. MTK, R0, AL, FH, AD, FTR, JB, PD, AM0, EA-L, JSB, DB, AF, M-CK, KD and MRS declare no conflict of interest.

\section{Patient consent for publication Not applicable.}

Ethics approval This study involves human participants and was approved by the Cancer Institute NSW Clinical Research Ethics Committee (2007C/10/027). Participants gave informed consent to participate in the study before taking part.

Provenance and peer review Not commissioned; externally peer reviewed. Data availability statement Data are available upon reasonable request.

Supplemental material This content has been supplied by the author(s). It has not been vetted by BMJ Publishing Group Limited (BMJ) and may not have been peer-reviewed. Any opinions or recommendations discussed are solely those of the author(s) and are not endorsed by BMJ. BMJ disclaims all liability and responsibility arising from any reliance placed on the content. Where the content includes any translated material, BMJ does not warrant the accuracy and reliability of the translations (including but not limited to local regulations, clinical guidelines, terminology, drug names and drug dosages), and is not responsible for any error and/or omissions arising from translation and adaptation or otherwise.

Open access This is an open access article distributed in accordance with the Creative Commons Attribution Non Commercial (CC BY-NC 4.0) license, which permits others to distribute, remix, adapt, build upon this work non-commercially, and license their derivative works on different terms, provided the original work is properly cited, an indication of whether changes were made, and the use is noncommercial. See: http://creativecommons.org/licenses/by-nc/4.0/. 


\section{Original research}

\section{ORCID iDs}

Yeh Chen Lee http://orcid.org/0000-0003-2009-8263

Jonathan A Ledermann http://orcid.org/0000-0003-3799-3539

Michael L Friedlander http://orcid.org/0000-0003-3090-795X

\section{REFERENCES}

1 Friedlander ML, Stockler M, O'Connell R, et al. Symptom burden and outcomes of patients with platinum resistant/refractory recurrent ovarian cancer: a reality check: results of stage 1 of the Gynecologic Cancer Intergroup Symptom Benefit Study. Int J Gynecol Cancer 2014;24:857-64.

2 Hwang $\mathrm{K}-\mathrm{H}$, Cho O-H, Yoo Y-S. Symptom clusters of ovarian cancer patients undergoing chemotherapy, and their emotional status and quality of life. Eur J Oncol Nurs 2016;21:215-22.

3 Friedlander M, Mercieca-Bebber RL, King MT. Patient-reported outcomes (PRO) in ovarian cancer clinical trials-lost opportunities and lessons learned. Ann Oncol 2016;27 Suppl 1:i66-71.

4 Wilson MK, Friedlander ML, Joly F, et al. A systematic review of health-related quality of life reporting in ovarian cancer phase III clinical trials: room to improve. Oncologist 2018;23:203-13.

5 Joly F, Hilpert F, Okamoto A, et al. Fifth Ovarian Cancer Consensus Conference of the Gynecologic Cancer InterGroup: recommendations on incorporating patient-reported outcomes in clinical trials in epithelial ovarian cancer. Eur $J$ Cancer 2017;78:133-8.

6 Stockler MR, Hilpert F, Friedlander M, et al. Patient-reported outcome results from the open-label phase III AURELIA trial evaluating bevacizumab-containing therapy for platinum-resistant ovarian cancer. J Clin Oncol 2014;32:1309-16.

7 Lindemann K, Gibbs E, Åvall-Lundqvist E, et al. Chemotherapy vs tamoxifen in platinum-resistant ovarian cancer: a phase III, randomised, multicentre trial (Ovaresist). Br J Cancer 2017:116:455-63.

8 Pujade-Lauraine E, Banerjee S, Pignata S. Management of platinum-resistant, relapsed epithelial ovarian cancer and new drug perspectives. J Clin Oncol 2019;37:2437-48.

9 Matulonis UA. Management of newly diagnosed or recurrent ovarian cancer. Clin Adv Hematol Oncol 2018;16:426-37.

10 Hanker LC, Loibl S, Burchardi N, et al. The impact of second to sixth line therapy on survival of relapsed ovarian cancer after primary taxane/platinum-based therapy. Ann Oncol 2012;23:2605-12.

11 Roncolato FT, Joly F, O'Connell R, et al. Reducing uncertainty: predictors of stopping chemotherapy early and shortened survival time in platinum resistant/refractory ovarian cancer-the GCIG Symptom Benefit Study. Oncologist 2017;22:1117-24.

12 Roncolato FT, O'Connell RL, Joly F, et al. Predictors of progression free survival, overall survival and early cessation of chemotherapy in women with potentially platinum sensitive (PPS) recurrent ovarian cancer (ROC) starting third or subsequent line $(\geq 3)$ chemotherapy - The GCIG Symptom Benefit Study (SBS). Gynecol Oncol 2020;156:45-53.

13 King MT, Stockler MR, O'Connell RL, et al. Measuring what matters most: validation of the measure of ovarian symptoms and treatment, a patient-reported outcome measure of symptom burden and impact of chemotherapy in recurrent ovarian cancer. Qual Life Res 2018;27:59-74.

14 King MT, Stockler MR, Butow P, et al. Development of the measure of ovarian symptoms and treatment concerns: aiming for optimal measurement of patient-reported symptom benefit with chemotherapy for symptomatic ovarian cancer. Int $J$ Gynecol Cancer 2014;24:865-73.

15 Aaronson NK, Ahmedzai S, Bergman B, et al. The European Organisation for Research and Treatment of Cancer QLQ-C30: a quality-of-life instrument for use in international clinical trials in oncology. J Natl Cancer Inst 1993;85:365-76.
16 Osoba D, Zee B, Pater J, et al. Psychometric properties and responsiveness of the EORTC quality of life questionnaire (QLQ-C30) in patients with breast, ovarian and lung cancer. Qual Life Res 1994;3:353-64.

17 Greimel E, Bottomley A, Cull A, et al. An international field study of the reliability and validity of a disease-specific questionnaire module (the QLQ-OV28) in assessing the quality of life of patients with ovarian cancer. Eur J Cancer 2003;39:1402-8.

18 Basen-Engquist K, Bodurka-Bevers D, Fitzgerald MA, et al. Reliability and validity of the functional assessment of cancer therapy-ovarian. J Clin Oncol 2001;19:1809-17.

19 Jensen SE, Rosenbloom SK, Beaumont JL, et al. A new index of priority symptoms in advanced ovarian cancer. Gynecol Oncol 2011;120:214-9.

20 Gundy CM, Fayers PM, Groenvold M, et al. Comparing higher order models for the EORTC QLQ-C30. Qual Life Res 2012;21:1607-17.

21 Giesinger JM, Kieffer JM, Fayers PM, et al. Replication and validation of higher order models demonstrated that a summary score for the EORTC QLQ-C30 is robust. J Clin Epidemiol 2016;69:79-88.

22 King MT, Costa DSJ, Aaronson NK, et al. QLU-C10D: a health state classification system for a multi-attribute utility measure based on the EORTC QLQ-C30. Qual Life Res 2016:25:625-36.

23 King MT, Viney R, Pickard AS, et al. Australian utility weights for the EORTC QLU-C10D, a multi-attribute utility instrument derived from the cancer-specific quality of life questionnaire, EORTC QLQ-C30. Pharmacoeconomics 2018;36:225-38.

24 Oskay-Özcelik G, Alavi S, Richter R, et al. Expression III: patients' expectations and preferences regarding physician-patient relationship and clinical management-results of the International NOGGO/ENGOT-ov4-GCIG study in 1830 ovarian cancer patients from European countries. Ann Oncol 2018;29:910-6.

25 Santini D, Armento G, Giusti R, et al. Management of orphan symptoms: ESMO clinical practice guidelines for diagnosis and treatment†. ESMO Open 2020;5.

26 Loprinzi CL, Lacchetti C, Bleeker J, et al. Prevention and management of chemotherapy-induced peripheral neuropathy in survivors of adult cancers: ASCO guideline update. J Clin Oncol 2020;38:3325-48.

27 Hay CM, Courtney-Brooks M, Lefkowits C, et al. Symptom management in women with recurrent ovarian cancer: do patients and clinicians agree on what symptoms are most important? Gynecol Oncol 2016;143:367-70.

28 Chandwani KD, Zhao F, Morrow GR, et al. Lack of patient-clinician concordance in cancer patients: its relation with patient variables. $J$ Pain Symptom Manage 2017;53:988-98.

29 Kotronoulas G, Kearney N, Maguire R, et al. What is the value of the routine use of patient-reported outcome measures toward improvement of patient outcomes, processes of care, and health service outcomes in cancer care? A systematic review of controlled trials. J Clin Oncol 2014;32:1480-501.

30 Cleeland CS, Wang XS, Shi Q, et al. Automated symptom alerts reduce postoperative symptom severity after cancer surgery: a randomized controlled clinical trial. J Clin Oncol 2011;29:994-1000.

31 Basch E, Deal AM, Kris MG, et al. Symptom monitoring with patientreported outcomes during routine cancer treatment: a randomized controlled trial. J Clin Oncol 2016;34:557-65.

32 Ishaque S, Karnon J, Chen G, et al. A systematic review of randomised controlled trials evaluating the use of patient-reported outcome measures (PROMs). Qual Life Res 2019;28:567-92.

33 Basch E, Deal AM, Dueck AC, et al. Overall survival results of a trial assessing patient-reported outcomes for symptom monitoring during routine cancer treatment. JAMA 2017;318:197-8.

34 Kurtz J-E, Gebski V, Sukhin V, et al. Incorporating patient centered benefits as endpoints in randomized trials of maintenance therapies in advanced ovarian cancer: a position paper from the GCIG Symptom Benefit Committee. Gynecol Oncol 2021;161:502-7. 